\title{
Perfil epidemiológico de pacientes portadores de insuficiência cardíaca atendidos em um hospital de referência no Norte do Brasil
}

\author{
Epidemiological profile of patients with heart failure attended at a reference hospital in \\ North Brazil
}
Perfil epidemiológico de pacientes con insuficiencia cardíaca atendidos en un hospital de referencia en el Norte de Brasil

Mauricio Soares Carneiro ${ }^{1 *}$, Helder José Lima Reis.

\begin{abstract}
RESUMO
Objetivo: Descrever as características sociodemográficas e clínicas de pacientes admitidos com diagnóstico de Insuficiência Cardíaca (IC) em um hospital público de referência no Norte do Brasil. Método Estudo observacional, transversal, descritivo, com caráter quantitativo, envolvendo 65 participantes internados em um hospital de referência com diagnóstico clínico de Insuficiência Cardíaca Congestiva (ICC) descompensada, no período de janeiro a dezembro de 2017. Foram incluídos no estudo, participantes maiores de 18 anos, que atingiram mais de 7 pontos nos critérios de Boston para diagnóstico da doença. Para comparar as causas de descompensação foram aplicados os testes do Quiquadrado e de Kruskal-Wallis por meio do programa BioEstat versão 5.3. Resultados: A mediana da idade dos 65 participantes foi de 64 anos, 56,9\% eram homens. Os principais fatores associados à IC foram hipertensão arterial $(66,2 \%)$, tabagismo $(70,8 \%)$ e sedentarismo (63,1\%). As principais etiologias foram cardiopatia hipertensiva (23\%), cardiopatia isquêmica, cardiomiopatia idiopática e doença valvar (18,5\% cada). As principais causas de descompensação observadas foram disfunção valvar $(26,1 \%)$ e infecção $(15,3 \%)$. Conclusão: A compreensão da doença, dos fatores associados, do tratamento, bem como o acesso aos serviços básicos da rede de saúde pública devem ser priorizados para a melhora da assistência na região Norte do país.
\end{abstract}

Palavras-chave: Insuficiência cardíaca, Diretrizes, Perfil epidemiológico.

\begin{abstract}
Objective: To describe the sociodemographic and clinical characteristics of patients admitted with a diagnosis of Heart Failure (HF) in a public reference hospital in North Brazil. Methods: This is an observational, cross-sectional, descriptive, quantitative study, involving 65 participants admitted to a reference hospital with a clinical diagnosis of descompensated Congestive Heart Failure (CHF), from January to December 2017. Were included in the study patients over 18-year-olds, who scored more than 7 points on the Boston criterion for diagnosing the disease. To compare the causes of decompensation, the Chi-square and Kruskal-Wallis tests were applied using the BioEstat version 5.3 program. Results: The median age of the 65 participants was 64 years, $56.9 \%$ were men. The main factors associated with HF were arterial hypertension $(66.2 \%)$, smoking $(70.8 \%)$ and sedentary lifestyle $(63.1 \%)$. The main etiologies were hypertensive heart disease $(23 \%)$, ischemic heart disease, idiopathic cardiomyopathy and valve disease (18.5\% each). The main causes of decompensation observed were valve dysfunction (26.1\%) and infection (15.3\%). Conclusion: Understanding the disease, associated factors, treatment, as well as access to basic services in the public health network should be prioritized to improve care in the northern region of the country.
\end{abstract}

Keywords: Heart failure, Guidelines, Epidemiological profile.

\section{RESUMEN}

Objetivo: Describir las características sociodemográficas y clínicas de los pacientes ingresados con diagnóstico de Insuficiencia Cardíaca (IC) en un hospital público de referencia en el Norte de Brasil. Métodos: Un estudio observacional, transversal, descriptivo, cuantitativo, que involucró a 65 participantes ingresados en un hospital de referencia con diagnóstico clínico de Insuficiencia Cardíaca Congestiva (ICC) descompensada, de enero a diciembre de 2017. Se incluyeron participantes mayores de 18 años, que obtuvo más de 7 puntos en los criterios de Boston para el diagnóstico de la enfermedad. Para comparar las causas de descompensación se aplicaron las pruebas de Chicuadrado y Kruskal-Wallis utilizando el programa BioEstat versión 5.3. Resultados: La mediana de edad de los 65 participantes fue de 64 años, el 56,9\% eran hombres. Los principales factores asociados a la IC fueron la hipertensión arterial $(66,2 \%)$, el tabaquismo $(70,8 \%)$ y el sedentarismo $(63,1 \%)$. Las principales etiologías fueron cardiopatía hipertensiva (23\%), cardiopatía isquémica, miocardiopatía idiopática y valvulopatía (18,5\% cada una). Las principales causas de descompensación observadas fueron disfunción valvular $(26,1 \%)$ e infección (15,3\%). Conclusión: Se debe priorizar el conocimiento de la enfermedad, los factores asociados, el tratamiento, así como el acceso a los servicios básicos en la red de salud pública para mejorar la atención en la región norte del país.

Palabras clave: Insuficiencia cardíaca, Guías, Perfil epidemiológico.

${ }^{1}$ Fundação Hospital de Clínicas Gaspar Viana, Belém - PA. *E-mail: mscarneiro@ufpa.br

SUBMETIDO EM: 11/2021 


\section{INTRODUÇÃO}

A insuficiência cardíaca (IC) se mantém como um importante problema de saúde pública mundial (ROHDE LEP, et al., 2018; SOUZA DSM e POVOA RMS, 2016). Um estudo realizado por McKee P, et al. (1971), iniciado em 1949, acompanhou mais de cinco mil pessoas sadias durante o período de dezesseis anos e demonstrou uma incidência para IC de 2,3/1000/ano entre homens e 1,4/1000/ano entre mulheres. Uma revisão sistematizada mais recente, estima a prevalência em cerca de 37 milhões de indivíduos no mundo (ZIAEIAN B e FONAROW GC, 2016).

Dados americanos apontam a existência de cerca de 5,7 milhões de pessoas acima dos 20 anos de idade com IC no ano de 2012, com cerca de 915.000 novos casos naquele ano em indivíduos acima dos 55 anos. Ocorreram nos EUA mais de 65.000 óbitos diretamente relacionados à IC em 2013 e o custo total com a doença em 2012 foi estimado em 30,7 bilhões de dólares (MOZAFFARIAN D, et al., 2016). Vale ressaltar que a mortalidade por IC vem caindo ao longo dos anos, porém ainda permanece alta do ponto de vista epidemiológico, gerando impactos para a saúde pública (GERBER Y, et al., 2015).

No Brasil, segundo o estudo de Stevens B, et al. (2018), em 2015 havia mais de 2,8 milhões de pessoas acima de vinte anos com IC, resultado que representa cerca de $2 \%$ da população adulta, e o custo da doença naquele ano atingiu cerca de 14,5 milhões de reais, ou pouco mais de 5 mil reais por caso. Albuquerque DC, et al. (2015), em estudo publicado referente ao "Estudo BREATHE", observaram que no ano de 2014 a mortalidade intra-hospitalar por IC foi de $12,6 \%$.

Apesar dos fatores de morbimortalidade relacionados à IC serem incontestáveis, os dados clínicos destes pacientes, bem como o impacto do gerenciamento do quadro sobre os resultados durante a internação, permanecem inconsistentes e incompletos, uma vez que grande parte das informações são oriundas de estudo isolados e estudos clínicos que não necessariamente representam a população de pacientes hospitalizados por essa patologia (ADAMS JR, et al., 2005; ROGER VL, 2013).

No Brasil, existem poucas publicações que avaliam de forma clara e prospectiva características sociodemográficas, clínicas e prognósticas de pacientes hospitalizados com diagnóstico clínico de IC. Alguns estudos, sugerem a existência de disparidades regionais significativas envolvendo diversas características dos pacientes que internam com essa doença no Brasil, mas estas comparações são metodologicamente limitadas por delineamentos e critérios de inclusão muitas vezes divergentes (STEWART S, et al., 2001; TAVARES LR, et al., 2002).

Dessa forma, acredita-se que o estabelecimento de um registro regional de pacientes de um hospital público da região norte, poderá retratar de forma mais precisa o perfil dos pacientes que internam com diagnóstico de IC, como estes pacientes são tratados e qual seu prognóstico em curto e longo prazo. Comparações regionais com outros estudos semelhantes também poderão ser viabilizadas. Neste contexto, o presente estudo objetivou analisar as características sociodemográficas e clínicas dos pacientes com diagnóstico de IC, atendidos em um hospital de referência no Norte do Brasil, com intuito de documentar de forma sistemática e efetiva, o perfil da população que busca assistência hospitalar, na rede pública, bem como a qualidade do atendimento prestado.

\section{MÉTODOS}

Trata-se de um estudo observacional, transversal, descritivo, com caráter quantitativo, envolvendo 65 participantes internados em um hospital de referência localizado na região Norte do Brasil, com diagnóstico clínico de Insuficiência Cardíaca Congestiva descompensada, no período de janeiro a dezembro de 2017. A população do presente estudo faz parte do I Registro Brasileiro de IC - sigla em inglês "BREATHE", em andamento desde fevereiro de 2011.

A coleta de dados foi realizada após 24h da admissão hospitalar, por meio de fichas desenvolvidas pelos investigadores do estudo BREATHE. Foram recrutados todos os participantes enquadrados nos critérios de inclusão do estudo e que assinaram previamente o Termo de Consentimento Livre e Esclarecido, anuindo assim sua participação na pesquisa. Foram definidos como critérios de inclusão: ter mais de 18 anos, atingir mais do que 7 pontos nos critérios de Boston para diagnóstico de IC e possuir no mínimo 24h de 
internação na instituição de saúde onde ocorreu a pesquisa. Foram excluídos do estudo os pacientes submetidos à angioplastia coronariana ou cirurgia de revascularização do miocárdio no último mês e pacientes com IC secundária a sepse (CARLSON KJ, et al., 1985).

A análise dos dados foi realizada por meio de métodos estatísticos descritivos e inferenciais. As variáveis quantitativas foram avaliadas utilizando-se medidas de tendência central e de variação. Já, as variáveis qualitativas foram analisadas por meio de distribuições de frequências absolutas e relativas. Para comparar as causas de descompensação foi aplicado o teste do Qui-quadrado nas variáveis qualitativas e o teste de Kruskal-Wallis, com pós-teste de Dunn, para comparar as variáveis quantitativas. Foi previamente fixado o nível de significância alfa $=0.05$ (margem de erro alfa de 5\%) para rejeição da hipótese de nulidade. $O$ processamento estatístico foi realizado no programa BioEstat versão 5.3 e o planejamento da análise de dados foi realizado na tecnologia Statistical Analysis Model (SAM) (AYRES M, et al., 2007).

Este estudo foi submetido e aprovado pelo Comitê de Ética em Pesquisa da Fundação Hospital de Clínicas Gaspar Viana, sob o parecer de número 1.623.947.

\section{RESULTADOS}

O presente estudo avaliou 65 pacientes que foram agrupados conforme as causas de descompensação mais frequentes: GA infecção $(15,3 \%) n=10$, GB causa valvar $(26,1 \%) n=17$ e GX (outras causas) $n=38$. Quanto aos aspectos sociodemográficos da amostra, observou-se as seguintes características predominantes, as quais tiveram $p$ valor $<0.05$, quando testadas sob a hipótese de serem presentes na maioria dos casos: raça parda (61.5\%), nível de escolaridade fundamental incompleto (53.8\%), renda familiar de até 1 salário mínimo (55.4\%) e idade variando entre 55 e 70 anos com mediana de 64 anos (Tabela 1 e Tabela 2).

Tabela 1- Caracterização dos participantes do estudo, conforme a causa da descompensação: GA (infecção, $n=10$ ), GB (causa valvar, $n=17$ ) e $G X$ (outras causas, $n=38$ ).

\begin{tabular}{|c|c|c|c|c|c|c|c|c|c|c|}
\hline \multirow{2}{*}{ Variáveis } & \multirow{2}{*}{$\begin{array}{c}\text { GA } \\
\mathrm{n} \\
\end{array}$} & \multicolumn{2}{|r|}{ GB } & \multicolumn{3}{|c|}{ GX } & \multicolumn{2}{|c|}{ Geral } & \multirow{2}{*}{$\begin{array}{c}\text { Diferença } \\
\text { p-valor }\end{array}$} & \multirow{2}{*}{$\begin{array}{c}\text { Tendência } \\
\text { p-valor }\end{array}$} \\
\hline & & $\%$ & $\mathrm{n}$ & $\%$ & $\mathrm{n}$ & $\%$ & $\mathrm{n}$ & $\%$ & & \\
\hline Etiologia & & & & & & & & & $0.0005^{\star}$ & 0.2733 \\
\hline Isquêmica & 1 & 10.0 & 3 & 17.6 & 8 & 21.1 & 12 & 18.5 & & \\
\hline Cardiomiopatia Idiopática & 2 & 20.0 & 2 & 11.8 & 8 & 21.1 & 12 & 18.5 & & \\
\hline Hipertensiva & 3 & 30.0 & 1 & 5.9 & 11 & 28.9 & 15 & 23.1 & & \\
\hline Doença de Chagas & 2 & 20.0 & 0 & 0.0 & 2 & 5.3 & 4 & 6.2 & & \\
\hline Doença Valvar* & 0 & 0.0 & 10 & 58.8 & 2 & 5.3 & 12 & 18.5 & & \\
\hline Outros & 2 & 20.0 & 1 & 5.9 & 7 & 18.4 & 10 & 15.4 & & \\
\hline Sexo & & & & & & & & & 0.1349 & 0.3211 \\
\hline Masculino & 8 & 80.0 & 11 & 64.7 & 18 & 47.4 & 37 & 56.9 & & \\
\hline Feminino & 2 & 20.0 & 6 & 35.3 & 20 & 52.6 & 28 & 43.1 & & \\
\hline Raça & & & & & & & & & 0.8778 & $<0.0001^{\star \star}$ \\
\hline Branca & 2 & 20.0 & 3 & 17.6 & 8 & 21.1 & 13 & 20.0 & & \\
\hline Negra & 3 & 30.0 & 3 & 17.6 & 6 & 15.8 & 12 & 18.5 & & \\
\hline Parda** & 5 & 50.0 & 11 & 64.7 & 24 & 63.2 & 40 & 61.5 & & \\
\hline Escolaridade & & & & & & & & & 0.9206 & $<0.0001^{\star \star}$ \\
\hline Analfabeto & 2 & 20.0 & 3 & 17.6 & 4 & 10.5 & 9 & 13.8 & & \\
\hline Fund Incompleto** & 4 & 40.0 & 10 & 58.8 & 21 & 55.3 & 35 & 53.8 & & \\
\hline Fund Completo & 1 & 10.0 & 1 & 5.9 & 4 & 10.5 & 6 & 9.2 & & \\
\hline Médio Incompleto & 1 & 10.0 & 1 & 5.9 & 4 & 10.5 & 6 & 9.2 & & \\
\hline Médio Completo & 2 & 20.0 & 2 & 11.8 & 2 & 5.3 & 6 & 9.2 & & \\
\hline Sup Incompleto & 0 & 0.0 & 0 & 0.0 & 2 & 5.3 & 2 & 3.1 & & \\
\hline Sup Completo & 0 & 0.0 & 0 & 0.0 & 1 & 2.6 & 1 & 1.5 & & \\
\hline Renda familiar & & & & & & & & & 0.8228 & $<0.0001^{\star *}$ \\
\hline Menor ou até $1 \mathrm{SM}^{* *}$ & 5 & 50.0 & 11 & 64.7 & 20 & 52.6 & 36 & 55.4 & & \\
\hline Entre 1 e 2 SM & 5 & 50.0 & 6 & 35.3 & 15 & 39.5 & 26 & 40.0 & & \\
\hline Entre 2 e 5 SM & 0 & 0.0 & 0 & 0.0 & 2 & 5.3 & 2 & 3.1 & & \\
\hline Mais que $10 \mathrm{SM}$ & 0 & 0.0 & 0 & 0.0 & 1 & 2.6 & 1 & 1.5 & & \\
\hline
\end{tabular}

Legenda: ${ }^{*}$ Qui-quadrado de independência. ${ }^{* *}$ Qui-quadrado de tendência.

Fonte: Carneiro MS e Reis HJL, 2021. 
A partir da análise estatística inferencial, tem-se que os fatores associados à IC nessa amostra foram: hipertensão arterial (66.2\%), tabagismo (70.8\%) e sedentarismo (63.1\%).Quando se fez a comparação entre as causas de descompensação revelaram-se dois fatores com real diferença: 1- a idade no GA (Infecção) teve mediana 49.5 anos, significativamente menor ( $p$-valor $=0.0441^{*}$ ) do que no GB (Causa Valvar) com mediana 63.0 anos e GX (outras causas) com mediana 65 anos; 2- entre as etiologias mais frequentes no estudo, doença valvar esteve presente em $58.8 \%$ dos 17 pacientes com descompensação por causa valvar, essa proporção foi significativamente diferente ( $p$-valor $=0.0005^{*}$ ), quando comparado com a proporção de doença valvar no GA $(0.0 \%)$ e GX (5.3\%). A presença de fatores associados à IC não apresentou diferença estatisticamente significativa conforme a causa da descompensação (Tabela 2 e Tabela 3).

Tabela 2 - Idade dos participantes, conforme a causa da descompensação: GA (infecção, n=10), GB (causa valvar, $n=17$ ) e $G X$ (outras causas, $n=38$ ).

\begin{tabular}{lcccc}
\hline Idade (anos) & GA & GB & GX & Geral \\
\hline Mediana & $\mathbf{4 9 . 5}$ & $\mathbf{6 3 . 0}$ & $\mathbf{6 5 . 0}$ & $\mathbf{6 4 . 0}$ \\
\hline 10. Quartil & 46.5 & 59.0 & 62.0 & 55.0 \\
3o. Quartil & 58.8 & 67.0 & 70.8 & 70.0 \\
Média & 51.8 & 60.5 & 64.3 & 61.4 \\
DP & 16.9 & 13.2 & 14.7 & 15.1 \\
\hline
\end{tabular}

Nota: ${ }^{*} p$-valor $=0.0441$, Teste de Kruskal-Wallis com pós-teste de Dunn.

Fonte: Carneiro MS e Reis HJL, 2021.

No tocante à idade (anos), no Geral $(n=65)$, apresentou mediana $=64$ anos com a variação interquartílica entre 55 e 70 anos. O GA apresentou menor mediana (49.5 anos) quando comparado com o GX (mediana $=65$ anos) e GB (mediana 63 anos).

Tabela 3 - Fatores associados conforme a causa da descompensação: GA (infecção, n=10), GB (causa valvar, $n=17$ ) e $G X$ (outras causas, $n=38$ ).

\begin{tabular}{|c|c|c|c|c|c|c|c|c|c|c|}
\hline \multirow{2}{*}{ Fatores de risco } & \multicolumn{2}{|l|}{ GA } & \multicolumn{2}{|l|}{ GB } & \multicolumn{2}{|l|}{ GX } & \multicolumn{2}{|c|}{ Geral } & \multirow{2}{*}{$\begin{array}{c}\text { Diferença } \\
\text { p-valor }\end{array}$} & \multirow{2}{*}{$\begin{array}{c}\text { Tendência } \\
\text { p-valor }\end{array}$} \\
\hline & $\mathbf{n}$ & $\%$ & $\mathbf{n}$ & $\%$ & $\mathbf{n}$ & $\%$ & $\mathbf{n}$ & $\%$ & & \\
\hline Hipertensão Arterial & 5 & 50.0 & 9 & 52.9 & 29 & 76.3 & 43 & 66.2 & 0.1198 & $0.0131^{* *}$ \\
\hline Dislipidemia & 2 & 20.0 & 6 & 35.3 & 11 & 28.9 & 19 & 29.2 & 0.6992 & \\
\hline Diabetes & 2 & 20.0 & 6 & 35.3 & 13 & 34.2 & 21 & 32.3 & 0.6621 & \\
\hline Doença Arterial Periférica & 2 & 20.0 & 0 & 0.0 & 5 & 13.2 & 7 & 10.8 & 0.2056 & \\
\hline Hist Familiar DAC & 2 & 20.0 & 5 & 29.4 & 7 & 18.4 & 14 & 21.5 & 0.6518 & \\
\hline Diagnóstico de IRC & 0 & 0.0 & 0 & 0.0 & 5 & 13.2 & 5 & 7.7 & 0.1501 & \\
\hline Diagnóstico Câncer & 0 & 0.0 & 0 & 0.0 & 0 & 0.0 & 0 & 0.0 & $\mathrm{n} / \mathrm{a}$ & \\
\hline AVC AIT prévio & 1 & 10.0 & 1 & 5.9 & 7 & 18.4 & 9 & 13.8 & 0.4286 & \\
\hline DPOC & 1 & 10.0 & 0 & 0.0 & 3 & 7.9 & 4 & 6.2 & 0.4645 & \\
\hline Depressão & 1 & 10.0 & 1 & 5.9 & 3 & 7.9 & 5 & 7.7 & 0.9251 & \\
\hline Sedentário & 6 & 60.0 & 12 & 70.6 & 28 & 73.7 & 46 & 70.8 & 0.6987 & $0.0013^{\star *}$ \\
\hline Tabagista ou Ex & 6 & 60.0 & 14 & 82.4 & 21 & 55.3 & 41 & 63.1 & 0.1534 & $0.0427^{* *}$ \\
\hline
\end{tabular}

Legenda: *Qui-quadrado de independência. ${ }^{* *}$ Qui-quadrado de tendência.

Fonte: Carneiro MS e Reis HJL, 2021.

Quanto aos achados laboratoriais encontrados neste estudo, a única variável encontrada com significância estatística foi a creatinina sérica, com mediana menor naqueles pacientes cuja causa da descompensação foi infecção, conforme demonstrado na Tabela 4. 
Tabela 4 - Variáveis laboratoriais conforme a causa da descompensação: GA (infecção, n=10), GB (causa valvar, $n=17$ ) e $G X$ (outras causas, $n=38$ ).

\begin{tabular}{|c|c|c|c|c|c|}
\hline Laboratoriais & GA & GB & GX & Geral & p-valor \\
\hline Glicemia & & & & & 0.1585 \\
\hline Mediana & 131.0 & 99.0 & 133.0 & 121.0 & \\
\hline 10. Quartil & 120.8 & 90.0 & 96.8 & 97.0 & \\
\hline 3o. Quartil & 140.5 & 118.0 & 167.3 & 144.0 & \\
\hline Média & 130.3 & 104.1 & 128.0 & 119.8 & \\
\hline DP & 22.7 & 21.8 & 51.9 & 40.0 & \\
\hline Creatinina & & & & & $0.0302^{*}$ \\
\hline Mediana & 0.92 & 1.01 & 1.26 & 1.12 & \\
\hline 10. Quartil & 0.88 & 0.90 & 1.08 & 0.92 & \\
\hline 3o. Quartil & 0.97 & 1.16 & 1.66 & 1.47 & \\
\hline Média & 0.95 & 1.06 & 1.57 & 1.33 & \\
\hline $\mathrm{DP}$ & 0.16 & 0.27 & 1.05 & 0.84 & \\
\hline Ureia & & & & & 0.7717 \\
\hline Mediana & 51.0 & 56.5 & 63.0 & 57.0 & \\
\hline 10. Quartil & 44.0 & 43.5 & 37.5 & 39.0 & \\
\hline 3o. Quartil & 74.0 & 73.5 & 91.0 & 86.5 & \\
\hline Média & 59.6 & 58.3 & 70.6 & 65.0 & \\
\hline $\mathrm{DP}$ & 20.9 & 23.0 & 40.9 & 33.3 & \\
\hline Sódio & & & & & 0.4018 \\
\hline Mediana & 131.0 & 135.5 & 135.0 & 134.5 & \\
\hline 10. Quartil & 130.0 & 132.5 & 132.0 & 131.0 & \\
\hline 3o. Quartil & 136.0 & 137.3 & 137.5 & 137.3 & \\
\hline Média & 130.6 & 135.2 & 132.2 & 132.7 & \\
\hline $\mathrm{DP}$ & 7.9 & 3.5 & 9.7 & 8.1 & \\
\hline Hemoglobina & & & & & 0.1095 \\
\hline Mediana & 14.7 & 14.3 & 12.4 & 13.2 & \\
\hline 10. Quartil & 12.2 & 12.8 & 11.1 & 12.1 & \\
\hline 3o. Quartil & 15.9 & 15.6 & 13.4 & 14.8 & \\
\hline Média & 23.5 & 14.1 & 12.6 & 14.9 & \\
\hline $\mathrm{DP}$ & 26.8 & 2.1 & 2.0 & 11.3 & \\
\hline Hematócrito & & & & & 0.2332 \\
\hline Mediana & 40.5 & 42.7 & 38.9 & 40.3 & \\
\hline 10. Quartil & 30.5 & 39.8 & 33.2 & 35.6 & \\
\hline 3o. Quartil & 48.2 & 49.3 & 41.2 & 47.0 & \\
\hline Média & 39.3 & 43.9 & 38.6 & 40.3 & \\
\hline $\mathrm{DP}$ & 10.5 & 6.5 & 6.9 & 7.7 & \\
\hline Potássio & & & & & 0.1972 \\
\hline Mediana & 3.85 & 4.10 & 4.12 & 4.05 & \\
\hline 10. Quartil & 3.75 & 3.88 & 3.85 & 3.80 & \\
\hline 3o. Quartil & 4.03 & 4.75 & 4.63 & 4.55 & \\
\hline Média & 3.93 & 4.31 & 4.37 & 4.26 & \\
\hline DP & 0.49 & 0.73 & 0.92 & 0.79 & \\
\hline
\end{tabular}

Nota: *Teste de Kruskal-Wallis com pós-teste de Dunn.

Fonte: Carneiro MS e Reis HJL, 2021.

Dentre os medicamentos para IC que vinham sendo utilizados pelos pacientes no momento da admissão hospitalar, não foram observadas diferenças estatisticamente significativas no uso conforme a causa da descompensação (Tabela 5). 
Tabela 5 - Medicamentos de uso domiciliar utilizados pelos participantes do estudo.

\begin{tabular}{|c|c|c|c|c|c|c|c|c|c|}
\hline \multirow{2}{*}{ Medic. Uso domiciliar } & \multicolumn{2}{|c|}{ GA } & \multicolumn{2}{|c|}{ GB } & \multicolumn{2}{|c|}{ GX } & \multicolumn{2}{|c|}{ Geral } & \multirow{2}{*}{$\begin{array}{c}\text { Diferença } \\
\text { p-valor }\end{array}$} \\
\hline & $\mathbf{n}$ & $\%$ & $\mathbf{n}$ & $\%$ & $\mathbf{n}$ & $\%$ & $\mathbf{n}$ & $\%$ & \\
\hline Antiagreganteplaquetário & 0 & 0.0 & 2 & 11.8 & 1 & 2.6 & 3 & 4.6 & 0.2473 \\
\hline Bloq Canais Cálcio & 1 & 10.0 & 1 & 5.9 & 4 & 10.5 & 6 & 9.2 & 0.8561 \\
\hline Diurético de Alça & 6 & 60.0 & 7 & 41.2 & 18 & 47.4 & 31 & 47.7 & 0.6382 \\
\hline Hidralazina & 0 & 0.0 & 0 & 0.0 & 0 & 0.0 & 0 & 0.0 & $\mathrm{n} / \mathrm{a}$ \\
\hline de ECA & 2 & 20.0 & 5 & 29.4 & 10 & 26.3 & 17 & 26.2 & 0.8651 \\
\hline Nitrato & 0 & 0.0 & 0 & 0.0 & 0 & 0.0 & 0 & 0.0 & $\mathrm{n} / \mathrm{a}$ \\
\hline Anticoagulante & 1 & 10.0 & 0 & 0.0 & 0 & 0.0 & 1 & 1.5 & $\mathrm{n} / \mathrm{a}$ \\
\hline Hipoglicemiante oral & 2 & 20.0 & 2 & 11.8 & 3 & 7.9 & 7 & 10.8 & 0.5404 \\
\hline Espironolactona & 5 & 50.0 & 7 & 41.2 & 11 & 28.9 & 23 & 35.4 & 0.3921 \\
\hline BloqReceptAngiotencina 2 & 3 & 30.0 & 3 & 17.6 & 9 & 23.7 & 15 & 23.1 & 0.7557 \\
\hline Ivabradina & 0 & 0.0 & 0 & 0.0 & 0 & 0.0 & 0 & 0.0 & $\mathrm{n} / \mathrm{a}$ \\
\hline Insulina & 0 & 0.0 & 0 & 0.0 & 1 & 2.6 & 1 & 1.5 & $\mathrm{n} / \mathrm{a}$ \\
\hline Beta bloqueador & 5 & 50.0 & 8 & 47.1 & 20 & 52.6 & 33 & 50.8 & 0.9283 \\
\hline Estatatina & 2 & 20.0 & 4 & 23.5 & 7 & 18.4 & 13 & 20.0 & 0.9087 \\
\hline Diruréticotiazídico & 0 & 0.0 & 0 & 0.0 & 0 & 0.0 & 0 & 0.0 & $\mathrm{n} / \mathrm{a}$ \\
\hline Digloxina & 1 & 10.0 & 4 & 23.5 & 3 & 7.9 & 8 & 12.3 & 0.2569 \\
\hline Amiodarona & 0 & 0.0 & 1 & 5.9 & 0 & 0.0 & 1 & 1.5 & $\mathrm{n} / \mathrm{a}$ \\
\hline
\end{tabular}

Nota: Teste aplicado - Qui-quadrado de independência.

Fonte: Carneiro MS e Reis HJL, 2021.

Quanto ao uso de medicamentos durante o período de internação, também não foram observadas diferenças na prescrição de medicamentos durante a internação hospitalar entre os grupos analisados conforme a causa de descompensação - infecção, causa valvar e outras (Tabela 6).

Tabela 6 - Medicamento de uso hospitalar utilizados pelos participantes do estudo.

\begin{tabular}{lccccccccc}
\hline \multirow{2}{*}{ Medic. uso hospitalar } & GA & \multicolumn{3}{c}{ GB } & \multicolumn{3}{c}{ GX } & \multicolumn{3}{c}{ Geral } & Diferença \\
\cline { 2 - 10 } & $\mathbf{n}$ & $\%$ & $\mathbf{n}$ & $\%$ & $\mathbf{n}$ & $\%$ & $\mathbf{n}$ & $\%$ & p-valor \\
\hline ANTI AGREGANTE PLAQUETÁRIO & $\mathbf{1}$ & $\mathbf{1 0 . 0}$ & $\mathbf{4}$ & $\mathbf{2 3 . 5}$ & $\mathbf{9}$ & $\mathbf{2 3 . 7}$ & $\mathbf{1 4}$ & $\mathbf{2 1 . 5}$ & $\mathbf{0 . 9 8 6 7}$ \\
\hline Aspirina & 0 & 0.0 & 1 & 5.9 & 2 & 5.3 & 3 & 4.6 & \\
Clopidogrel & 1 & 10.0 & 2 & 11.8 & 8 & 21.1 & 11 & 16.9 & \\
Prasugrel & 0 & 0.0 & 0 & 0.0 & 0 & 0.0 & 0 & 0.0 & \\
Ticagrelor & 0 & 0.0 & 0 & 0.0 & 0 & 0.0 & 0 & 0.0 & \\
Ticlopidina & 0 & 0.0 & 0 & 0.0 & 0 & 0.0 & 0 & 0.0 & \\
Outros & 0 & 0.0 & 1 & 5.9 & 0 & 0.0 & 1 & 1.5 & \\
\hline ANTICOAGULANTE & $\mathbf{6}$ & $\mathbf{6 0 . 0}$ & $\mathbf{5}$ & $\mathbf{2 9 . 4}$ & $\mathbf{2 0}$ & $\mathbf{5 2 . 6}$ & $\mathbf{3 1}$ & $\mathbf{4 7 . 7}$ & $\mathbf{0 . 1 9 6 5}$ \\
\hline Heparina N Fracionada & 0 & 0.0 & 0 & 0.0 & 1 & 2.6 & 1 & 1.5 & \\
Apixaban & 0 & 0.0 & 0 & 0.0 & 0 & 0.0 & 0 & 0.0 & \\
Enoxaparina & 6 & 60.0 & 5 & 29.4 & 18 & 47.4 & 29 & 44.6 & \\
Rivaroxaban, Fondaparinux & 0 & 0.0 & 0 & 0.0 & 0 & 0.0 & 0 & 0.0 & \\
Dabigatran, Warfaren, Endoxaban & 0 & 0.0 & 0 & 0.0 & 0 & 0.0 & 0 & 0.0 & \\
\hline BETA BLOQUEADOR & $\mathbf{7}$ & $\mathbf{7 0 . 0}$ & $\mathbf{9}$ & $\mathbf{5 2 . 9}$ & $\mathbf{2 2}$ & $\mathbf{5 7 . 9}$ & $\mathbf{3 8}$ & $\mathbf{5 8 . 5}$ & $\mathbf{0 . 6 8 1 9}$ \\
\hline Atenolol & 1 & 10.0 & 1 & 5.9 & 2 & 5.3 & 4 & 6.2 & \\
Propranolol & 0 & 0.0 & 0 & 0.0 & 0 & 0.0 & 0 & 0.0 & \\
Carvedilol & 6 & 60.0 & 8 & 47.1 & 18 & 47.4 & 32 & 49.2 & \\
Metoprolol & 0 & 0.0 & 0 & 0.0 & 1 & 2.6 & 1 & 1.5 & \\
Bisoprolol & 0 & 0.0 & 0 & 0.0 & 0 & 0.0 & 0 & 0.0 & \\
Nebivolol & 0 & 0.0 & 0 & 0.0 & 0 & 0.0 & 0 & 0.0 & \\
\hline BLOQ CANAIS CÁLCIO & $\mathbf{1}$ & $\mathbf{1 0 . 0}$ & $\mathbf{0}$ & $\mathbf{0 . 0}$ & $\mathbf{6}$ & $\mathbf{1 5 . 8}$ & $\mathbf{7}$ & $\mathbf{1 0 . 8}$ & $\mathbf{0 . 2 1 7 1}$ \\
\hline Nifedipina & 0 & 0.0 & 0 & 0.0 & 3 & 7.9 & 3 & 4.6 & \\
Diltiazen & 0 & 0.0 & 0 & 0.0 & 0 & 0.0 & 0 & 0.0 & \\
Amlodipina & 1 & 10.0 & 0 & 0.0 & 2 & 5.3 & 3 & 4.6 & \\
Verapamil & 0 & 0.0 & 0 & 0.0 & 0 & 0.0 & 0 & 0.0 & \\
\hline ESPIRONOLACTONA & 6 & $\mathbf{6 0 . 0}$ & $\mathbf{7}$ & $\mathbf{4 1 . 2}$ & $\mathbf{1 3}$ & $\mathbf{3 4 . 2}$ & $\mathbf{2 6}$ & $\mathbf{4 0}$ & $\mathbf{0 . 3 3 1 7}$ \\
\hline Ivabradina & 0 & 0.0 & 0 & 0.0 & 0 & 0.0 & 0 & 0.0 & n/a \\
Digoxina & 1 & 10.0 & 1 & 5.9 & 2 & 5.3 & 4 & 6.2 & 0.8562 \\
\hline & & & & & & & & \\
\hline
\end{tabular}




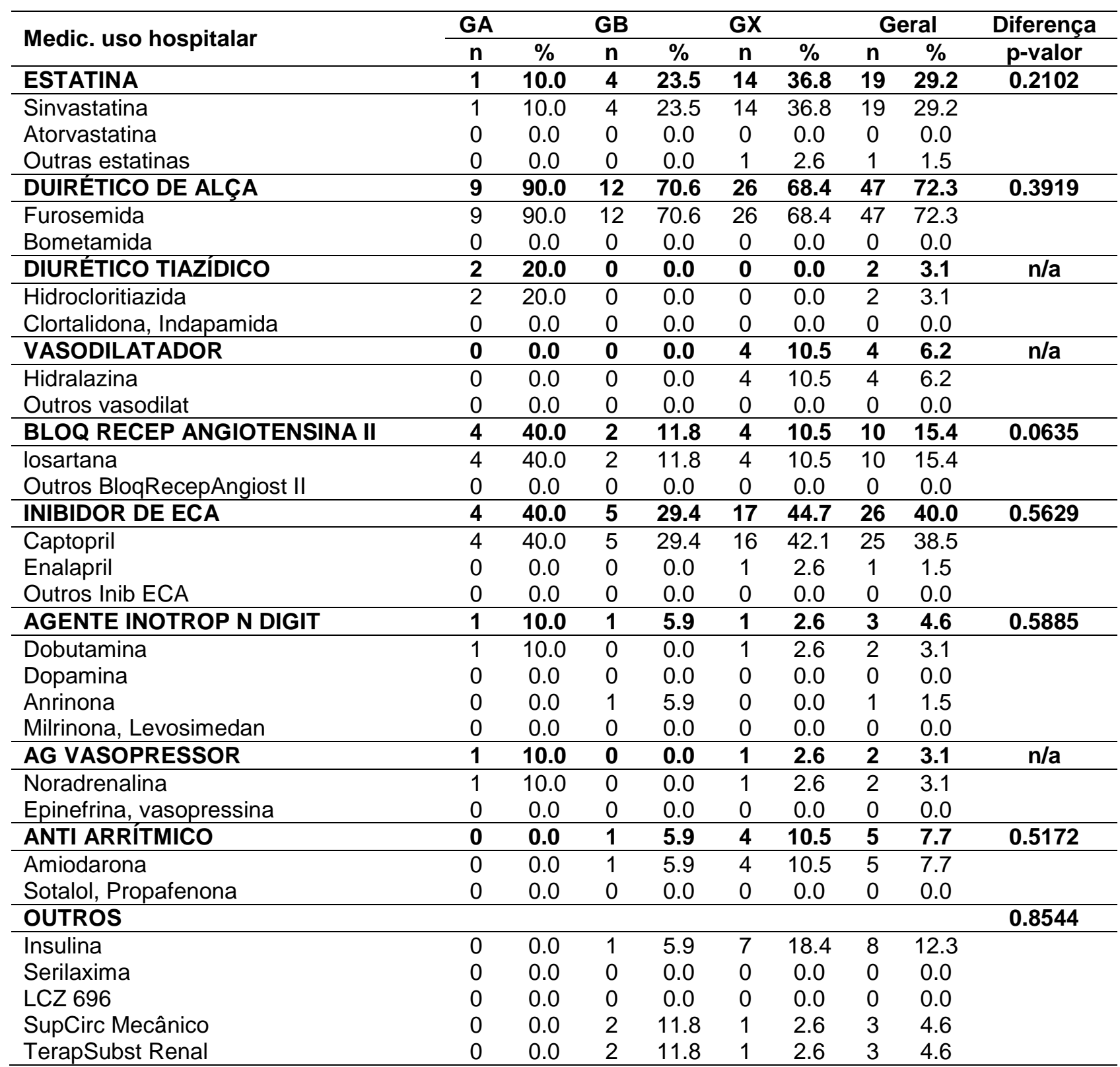

Nota: Teste aplicado: Qui-quadrado de independência. Fonte: dados do autor; Outros BloqRecepAngiost II: Valsartan, Ibesartan, Telmizartan, Candesartan, Omesartan; Outros Inib ECA: Lisinopril, Ramipril, Peridopril, Benazepril; Outros vasodilat: Nitrato, Nitroglicerina Intravenosa, Nitroprussiato; Outras estatinas: Atorvastatina, Rosuvastatina, Pravastatina e Fluvastatina

Fonte: Carneiro MS e Reis HJL, 2021.

\section{DISCUSSÃO}

Os principais achados sociodemográficos nessa amostra de 65 pacientes hospitalizados com IC descompensada foram, o predomínio da raça parda com $61,5 \%$ dos pacientes; um nível de escolaridade baixo caracterizado pelo predomínio do ensino fundamental incompleto $(53,8 \%)$; renda familiar mensal baixa, apenas um salário mínimo para $55,4 \%$ dos indivíduos e idade variando entre 55 e 70 anos com mediana de 64 anos. Este resultado corrobora os dados de Albuquerque DC, et al. (2015), onde a idade média dos participantes também foi de 64 anos, entretanto, os mesmos autores apontam o predomínio da etnia branca ( $59 \%$ dos indivíduos) em detrimento da parda, e que a idade média foi mais avançada no sul e sudeste e mais baixa na região norte.

O estudo conhecido por "EPICANiterói", realizado por Tavares LR et al. (2004), comparando o atendimento de IC em serviços público e privado no Brasil, demonstrou faixa etária inferior no serviço 
público. A média de idade foi acentuadamente diferente, havendo uma década de diferença entre os pacientes de serviços da rede pública e de hospitais privados, sendo que no serviço público encontravamse mais analfabetos e pessoas com renda familiar de até 1 salário-mínimo. O Registro Congestão estudou 143 pacientes em hospital privado em uma capital do nordeste. A média de idade dos pacientes foi de 72,40 $\pm 15,01$ anos, com $76,92 \%$ acima dos 61 anos e $54,55 \%$ homens. A maioria dos pacientes eram de etnia branca autorreferida $(45,45 \%)$, com ensino médio completo $(32,87 \%)$ e renda familiar maior que dois salários mínimos e menor ou igual a cinco salários mínimos (30,77\%) (SILVEIRA JRS, 2017).

Acredita-se, que essas diferenças regionais e entre pacientes provenientes do SUS e da iniciativa privada, caracterizando uma associação de baixa escolaridade e baixo poder aquisitivo com idade mais baixa de pacientes internados com IC, possivelmente refletem desigualdades no acesso ao sistema público de saúde e menor desenvolvimento socioeconômico na região (TAVARES LR, et al., 2014). Dessa forma, combater essas iniquidades em saúde, principalmente as que dizem respeito aos aspectos regionais, poderia promover a redução da incidência da IC no Brasil, bem como um acesso mais equânime à assistência em saúde. O presente estudo não analisou pacientes originados da rede privada.

Os estudos internacionais corroboram a associação entre desenvolvimento socioeconômico alto e idade mais elevada de pacientes internados por IC, como por exemplo, os realizados nos Estados Unidos e Europa, onde observou-se em todos idade média >70anos (ADAMS JR, et al., 2005; O'CONNOR CM, et al., 2008; NIEMINEN MS, et al., 2006; MAGGIONI AP, et al., 2010). Provavelmente, maiores poder aquisitivo e escolaridade produzem um melhor nível informacional dos pacientes e consequentemente uma adesão mais eficiente ao tratamento ambulatorial, postergando assim uma possível internação, fato que difere da população avaliada no presente estudo.

Um estudo tipo registro, que incluiu 1.232 pacientes internados por IC, realizado na India (Trivandrum HF Registry) por Harikrishnan S, et al. (2015), país tido como em desenvolvimento como o Brasil do chamado bloco BRICs (Brasil, Rússia, India, China), apresentou resultados semelhantes aos da presente pesquisa. A idade média foi de 61 anos e $57 \%$ dos pacientes tinham nível de escolaridade primário incompleto. Outro estudo que envolveu 8 países da Ásia e da região do Oceano Pacífico, associou a idade média dos pacientes internados devido a IC ao Índice de Desenvolvimento Humano dos diferentes países e ao estágio de transição epidemiológica, observando que em regiões menos desenvolvidas a idade dos pacientes tende a ser mais baixa (ATHERTON JJ, et al., 2012).

Analisando as etiologias de IC, observa-se como mais frequentes neste estudo a Hipertensão arterial sistêmica (HAS) (23\%), seguida dos casos de isquemia miocárdica, cardiomiopatia idiopática e doença valvar, todas com $18,5 \%$ dos casos avaliados. Esses dados corroboram resultados parciais de outro estudo realizado no Brasil, onde na região norte também predominou a HAS como principal etiologia de IC com $37 \%$ dos casos, enquanto que a isquemia predominou no sul, sudeste e nordeste (ALBUQUERQUE DC, et al., 2015). Grandes registros internacionais apontam para a presença de HAS e doença coronariana na história clínica de pacientes com IC (CRESPO-LEIRO MG, et al., 2016; YANCY CW, et al., 2013; ADAMS JR, et al., 2005).

Diferente do que se observa por meio dos resultados de pesquisas desenvolvidas com populações de países desenvolvidos, foi evidenciada uma elevada prevalência de doença valvar neste estudo. Já no registro indiano, sua presença é também considerável e atribuída a Doença Reumática, entidade ainda bastante presente nos países em desenvolvimento e mesmo em regiões pobres de países com elevado padrão de vida (HARIKRISHNAN S, et al., 2015; CARAPETIS JR, 2007). Em um registro intercontinental envolvendo 5.823 pacientes da África, Oriente Médio, Sudeste asiático, China e América do Sul também se observou incidência elevada de doença valvar (DOKAINISH H, et al., 2017). É razoável admitir que como no estudo indiano, nesta pesquisa a doença reumática tenha participação como causa de IC de etiologia valvar, embora não tenha sido objeto desse registro.

Os principais fatores associados à IC no presente estudo foram o sedentarismo para $70 \%$, hipertensão arterial para $66 \%$ e tabagismo para $63 \%$ dos pacientes. Revisando a literatura observa-se que os estudos não relatam a presença do sedentarismo nas amostras analisadas, possivelmente por não ser considerado 
uma comorbidade ou antecedente clínico, entretanto isto de forma nenhuma inviabiliza este resultado. A hipertensão está presente na história clínica de mais de $60 \%$ dos pacientes nos principais estudos sobre o perfil de pacientes hospitalizados com IC. O tabagismo foi relatado também como um fator estatisticamente significante (SULAIMAN K, et al., 2015; CRESPO-FILHO MG, et al., 2016; HARIKRISHNAN S, et al., 2015; PONIKOWSKI P, et al., 2014).

Ao analisar-se as causas de descompensação dos pacientes duas se destacaram, a disfunção valvar em $26 \%$ dos casos e infecção em $15 \%$. Os outros $59 \%$ foram representados por uma miscelânea de causas. Albuquerque DC, et al. (2015), observaram que a infecção foi a segunda principal causa de descompensação da IC (22\% dos casos) e a alteração valvar aguda esteve presente em $6,6 \%$ dos casos. $O$ elevado percentual de pacientes descompensados por alteração valvar aguda é semelhante ao relatado no estudo EFHS II, o qual apontou este quadro em 26,8\% dos participantes (NIEMINEN MS, et al., 2006). Outros importantes estudos também corroboram os resultados da presente pesquisa no tocante ao percentual de pacientes descompensados devido à instalação de processos infecciosos, como o GULF CARE, onde $15 \%$ dos pacientes descompensaram e o ALARM HF, onde essa frequência foi de $16 \%$ (SULAIMAN K, et al., 2015; FOLLATH F, et al., 2011)

O achado significativo de que os pacientes descompensados por infecção eram mais jovens não encontra paralelo na literatura consultada e os dados de que dispomos não permitem conclusões, devendo ser objeto de análise em outros estudos posteriores. $O$ fato de disfunção valvar ser observada de maneira bastante significativa como causa de descompensação de pacientes com doença valvar prévia como etiologia de IC pode refletir diagnóstico tardio e/ou acompanhamento inadequado das doenças valvares na amostra estudada, entretanto tal hipótese merece atenção especial e análise detalhada e caso comprovada que sirva de subsídio para tomadas de decisão que dirimam essa situação.

Observou-se que os pacientes admitidos devido a IC descompensada vinham subutilizando medicações de uso domiciliar recomendadas pelas diretrizes brasileiras e internacionais que comprovadamente reduzem a mortalidade por IC. Dentre as três principais classes de medicamentos recomendados $50 \%$ dos pacientes vinha utilizando beta- bloqueador, $49 \%$ em uso de Inibidor da Enzima de Conversão da Aldosterona (IECA), ou alternativamente usando Bloqueador dos Receptores da Angiotensina II (BRA) e 35\% estavam em uso de antagonista mineralocorticóide (espironolactona).

Diuréticos de alça vinham sendo utilizados no domicílio por cerca de $48 \%$ dos pacientes. Comparandose com estudos recentes, esses números são consideravelmente inferiores aos do estudo Trivandrum e ao estudo INTER-CHF com exceção da utilização de beta-bloqueador na África que foi de $48 \%$ (HARIKRISHNAN S et al., 2015; DOKAINISH H, et al., 2017). No estudo ESC-HF, dos pacientes admitidos com IC cerca de 60\% tomavam beta-bloqueador + IECA/BRA, 35\% utilizavam espironolactona e cerca de $65 \%$ estavam em uso de diurético. Pelo desenho do presente estudo não se pode afirmar se a subutilização de medicamentos recomendados por evidências científicas se deu pela não prescrição anterior, por má aderência ao tratamento, por ambos ou por dificuldade de acesso ao sistema de saúde, levando a admissão hospitalar sem adequado tratamento ambulatorial Pilot (MAGGIONI AP, et al., 2010).

Ao analisar as primeiras 24 horas de internação hospitalar observou-se que a prescrição de medicamentos recomendados pelas diretrizes ficou abaixo do desejável. Beta-bloqueador foi prescrito para $58,5 \%$ dos pacientes, IECA/ BRA II para cerca de $45 \%$ e apenas $40 \%$ dos pacientes foram prescritos com espironolactona. No ESC-HF Pilot, durante a internação, mais de $90 \%$ dos pacientes receberam IECA/ BRA, $86 \%$ receberam beta-bloqueador e $43,7 \%$ espironolactona, porém os autores ratificam que ainda há uma diferença entre as informações geradas pelos estudos randomizados, controlados e aquelas em geral observadas nas pesquisas que refletem a prática clínica (MAGGIONI AP, et al., 2010).

Publicado em 2017, o estudo QUALIFY incluiu mais de 6.600 pacientes com IC de todos os continentes e concluiu que a boa adesão dos médicos às diretrizes, prescrevendo pelo menos $50 \%$ das doses recomendadas de IECA/BRA, BBs, espironolactona e ivabradina, está diretamente associada à melhores resultados em seis meses, com redução significativa de taxas de hospitalização e mortalidade por IC e 
outras doenças cardiovasculares (KOMAJDA M, et al., 2017). Da mesma forma, o estudo IMPROVE-HF mostrou ao longo de 24 meses de acompanhamento que a melhora na adesão médica às práticas recomendadas por evidências tem associação positiva com a melhora da sobrevida (FONAROW GG, et al., 2010).

Vale ressaltar que uma limitação deste estudo está relacionada a amostragem, uma vez que apenas 65 participantes se enquadraram nos critérios de inclusão durante seu período de realização. Sugere-se assim, a realização de outros estudos que abordem esta temática na região Norte do Brasil, uma vez que a carência de dados científicos nesta região é um fator evidenciado e que pode comprometer diretamente a qualidade da assistência prestada.

\section{CONCLUSÃO}

A amostra caracterizou-se por pacientes com idade inferior à maioria dos estudos publicados, além de indivíduos que apresentam nível de escolaridade e renda familiar baixos. A Hipertensão arterial sistêmica, o tabagismo e o sedentarismo destacam-se entre os fatores associados à IC. Ao serem admitidos, esses pacientes vêm utilizando em percentual baixo os principais medicamentos recomendados para tratamento da IC. Com 24h de internação a taxa de prescrição de terapia adequada ainda permanece abaixo do desejável. Possíveis falhas por parte dos profissionais de saúde no esclarecimento quanto à gravidade e evolução progressiva da condição clínica e sobre a importância do estilo de vida devem ser consideradas. Há que se implementar estratégias para melhorar a taxa de adesão médica às diretrizes para tratamento da IC.

\section{REFERÊNCIAS}

1. ADAMS JR, et al. Characteristics and outcomes of patients hospitalized for heart failure in the United States: rationale, design, and preliminary observations from the first 100,000 cases in the Acute Decompensated Heart Failure National Registry (ADHERE). American Heart Journal, 2005; 149(2): 209-216.

2. ALBUQUERQUE DC, et al. I Registro Brasileiro de Insuficiência Cardíaca: aspectos clínicos, qualidade assistencial e desfechos hospitalares. Arquivos Brasileiros de Cardiologia, 2015; 104(6): 433-442.

3. ATHERTON JJ, et al. Patient characteristics from a regional multicenter database of acute decompensated heart failure in Asia Pacific (ADHERE International-Asia Pacific). Journal of Cardiac Failure, 2012; 18(1): 82-88.

4. AYRES M, et al. Aplicações estatísticas nas áreas das ciências bio-médicas. São Paulo: Instituto Mamirauá, 2007; $364 \mathrm{p}$.

5. CARAPETIS JR. Rheumatic heart disease in developing countries. New England Journal of Medicine, 2007; 357(5): 439-441.

6. CARLSON KJ, et al. An analysis of physicians: reasons for prescribing long-term digitalis therapy in outpatients. Journal of Clinical Epidemiology, 1985; 38(9): 733-739.

7. CRESPO-LEIRO MG, et al. European Society of Cardiology Heart Failure Long-Term Registry (ESC-HF-LT): 1-year follow-up outcomes and differences across regions. European Journal of Heart Failure, 2016; 18(6): 613-625.

8. DOKAINISH H, et al. Global mortality variations in patients with heart failure: results from the International Congestive Heart Failure (INTER-CHF) prospective cohort study. The Lancet Global Health, 2017; 5(7): 665-672.

9. FOLLATH F, et al. Clinical presentation, management and outcomes in the acute heart failure global survey of standard treatment (ALARM-HF). Intensivecare Medicine, 2011; 37(4): 619-626.

10. FONAROW GC, et al. Improving evidence-based care for heart failure in outpatient cardiology practices: primary results of the Registry to Improve the Use of Evidence-Based Heart Failure Therapies in the Outpatient Setting (IMPROVE HF). Circulation, 2010; 122(6): 585-596.

11. GERBER Y, et al. A contemporary appraisal of the heart failure epidemic in Olmsted County, Minnesota, 2000 to 2010.JAMA Internal Medicine, 2015; 175(6): 996-1004.

12. HARIKRISHNAN S, et al. Clinical presentation, management, in-hospital and 90-day outcomes of heart failure patients in Trivandrum, Kerala, India: the Trivandrum Heart Failure Registry. European Journal of Heart Failure, 2015; 17(8): 794-800.

13. KOMAJDA M, et al. Physicians' guideline adherence is associated with better prognosis in outpatients with heart failure with reduced ejection fraction: the QUALIFY international registry. European Journal of Heart Failure, 2017; 19(11): 1414-1423. 
14. MAGGIONI AP, et al. EURO Observational research programme: the heart failure pilot survey (ESC-HF Pilot). European Journal of Heart Failure, 2010; 12(10): 1076-1084.

15. MCKEE PA, et al. The natural history of congestive heartfailure: the Framingham study. New England Journal of Medicine, 1971; 285(26): 1441-1446.

16. MOZAFFARIAN D, et al. Executive summary: heart disease and stroke statistics-2016 update: a report from the American Heart Association. Circulation, 2016; 133(4): 447-454.

17. NIEMINEN MS, et al. Euro Heart Failure Survey II (EHFS II): a survey on hospitalized acute heart failure patients: description of population. European Heart Journal, 2006; 27(22): 2725-2736.

18. O'CONNOR CM, et al. Predictors of mortality after discharge in patients hospitalized with heart failure: an analysis from the Organized Program to Initiate Lifesaving Treatment in Hospitalized Patients with Heart Failure (OPTIMIZEHF). American Heart Journal, 2008; 156(4): 662-673.

19. PONIKOWSKI P, et al. Heart failure: preventing disease and death worldwide. ESC Heart Failure, 2014; $18(1)$ : 4-25.

20. ROGER VL. Epidemiology of heart failure. Circulation Research, 2013; 113(6): 646-659.

21. ROHDE LEP, et al. Diretriz Brasileira de Insuficiência Cardíaca Crônica e Aguda. Arquivos Brasileiros de Cardiologia, 2018; 111(3): 436-539.

22. SILVEIRA, JRS. Perfil sociodemográfico e econômico dos pacientes admitidos em hospital privado com insuficiência descompensada. Monografia (Graduação em Medicina) - Departamento de Medicina. Universidade Federal de Sergipe, Aracaju, 2017; 222-229p.

23. SOUZA DSM, POVOA RMS. Aspectos epidemiológicos e clínicos da doença de chagas aguda no Brasil e na América Latina. Revista da Sociedade de Cardiologia, 2016; 26(4): 222-229.

24. STEWART S, et al. Poles apart, but are they the same? A comparative study of Australian and Scottish patients with chronic heart failure. European Journal of Heart Failure, 2001; 3(2):249-255.

25. STEVENS B, et al. Os Custos das Doenças Cardíacas no Brasil. Arquivos Brasileiros de Cardiologia, 2018; 111(1): 29-36.

26. SULAIMAN K, et al. Clinical characteristics, management, and outcomes of acute heart failure patients: observations from the Gulf acute heart failure registry (Gulf CARE). European Journal of Heart Failure, 2015; 17(4): $374-384$.

27. TAVARES LR, et al. Epidemiologia da insuficiência cardíaca descompensada em Niterói: Projeto EPICANiterói. Arquivos Brasileiros de Cardiologia, 2004; 82(2): 121-124.

28. TAVARES LR, et al. Comorbidades e fatores de descompensação dos pacientes internados por insuficiência cardíaca descompesada na cidade de Niterói. Arquivos Brasileiros Cardiologia, 2002; 79(4): 35.

29. YANCY CW, et al. 2013 ACCF/AHA guideline for the management of heart failure: executive summary: a report of the American College of Cardiology Foundation/American Heart Association Task Force on practice guidelines. Journal of the American College of Cardiology, 2013; 62(16): 1495-1539.

30. ZIAEIAN B, FONAROW GC. Epidemiology and aetiology of heart failure. Nature Reviews Cardiology, 2016; 13(6): 368. 